\title{
EL "YO” EN HUSSERL, MOTIVO PARA UNA FENOMENOLOGÍA EN COLOMBIA
}

\author{
Juan Manuel Cuartas R. \\ Universidad del Valle
}

Recibido: marzo 7 de 2005

Aprobado: mayo 6 de 2005

\section{Resumen}

Cuando proponemos el "yo" en E. Husserl como horizonte común de los intereses filosóficos de Danilo Cruz Vélez y Daniel Herrera Restrepo, estamos recayendo sobre el problema que ha motivado las más diversas investigaciones en la historia de la filosofía, problema que puede reconocerse igualmente como primordial en la obra del filósofo alemán. El punto de partida será, por supuesto, ese "yo puro" que da ocasión a Husserl para señalar los pormenores de la correlación sujeto-objeto, concebida por él como el problema central de la fenomenología. Además de la profunda resonancia que D. Cruz Vélez y D. Herrera han conseguido dar a la obra de Husserl en nuestro medio, ¿a qué estamos tentados cuando intentamos poner en situación su trabajo en relación con el problema del "yo"? Más allá de los elogios y reconocimientos a su labor, la presente exposición se propone elevar una consulta en tomo a las afinidades y diversidades de las exégesis filosóficas.

\section{Palabras clave}

Danilo Cruz Vélez, Daniel Herrera Restrepo, yo trascendental, fenomenología.

\section{Abstract}

When we put forward the 'self' in E. Husserl as common horizon for the philosophic interests of Danilo Cruz Velez and Daniel Herrera Restrepo, we are falling back into the problem that has given rise to the most diverse investigations in the history of philosophy, issue that can be acknowledged as basic in the work of the german philosopher. The starting point would be, of course, 'pure self' that gives the occasion to Husserl to point out the details of the correlation between subject-object as conceived by him as the central issue of phenomenology. Besides the profound echo that D. Cruz Velez and D. Herrera 
have managed to give to Husserl's work in our context, ¿¿what are we tempted to, when we try to put in context his work in relation to the problem of the 'self'?. Further on the praises and acknowledgements to his work, the present exposing aims to boost the opinion around the affinities and diversities of the philosophic exegesis.

\section{Key words}

Danilo Cruz Vélez, Daniel Herrera Restrepo, trascendentales ich, phenomenology.

El hombre (así como todo ser finito) sólo entre hombres llega a ser hombre: y puesto que no puede ser otra cosa que hombre, y no sería en absoluto si no lo fuera, si ha de darse el hombre, en general, entonces tiene que ser varios.

Johann Gottlieb Fichte

\section{Preludio}

La comprensión adecuada de los problemas de la mente indaga tanto por las manifestaciones de lo mental como por lo mental mismo en su dinámica con el cuerpo. En este marco de acción la reflexión filosófica restituye uno de sus problemas fundamentales: el problema del yo. Cuando se concede, como lo hace el filósofo alemán Edmund Husserl (1859-1938), que el yo está en el mundo, que su motivación y su lenguaje pertenecen al mundo, se toma partido por un tipo de indagación no reduccionista que pone de precedente una lectura integrada del mundo interior y del mundo exterior humano. Estudiando el yo estudiamos la condición de pregunta como se instala el hombre en el mundo. Y en tiempos más recientes, todo lo que las ciencias cognitivas han conseguido adelantar en sus descripciones de lo mental, así como las neurociencias en su experimentación directa con los cerebros, son insumos que permiten advertir las derivaciones del problema del yo. En estos términos se propone además otro problema no menos definitivo de la filosofía: el del conocimiento.
Así, si la revitalización de estos dos tipos de problemas aparentemente realizan la subjetividad como motor, como valoración del ser y de su estar en el mundo, queda claro que un problema no descalifica al otro.

Evidentemente al retomar una noción tan clásica y controvertida como el yo, estamos volviendo sobre los presupuestos estrictamente filosóficos que lo señalaron en su momento como "yo analítico substante”, "yo sintético trascendental”, "yo puro”, etc. El espíritu de la fenomenología, que se expresa en los términos de la correlación sujeto-objeto, nos mueve a indagar la experiencia humana y a advertir cada vez con mayor claridad de qué manera está el sujeto en el mundo, de qué manera sus manifestaciones, organizaciones, usos y abusos derivan y repercuten en esa correlación. Poniendo en evidencia la amplitud de la tarea fenomenológica, que parte del reconocimiento, no de la negación, de la penetrabilidad y coextensionalidad del sujeto en el objeto, $y$ viceversa, el filósofo colombiano Daniel Herrera Restrepo nos recuerda siempre que: “en 1898 [Husserl] tuvo la intuición de que 
entre el hombre y el mundo existe una correlación”; así, el tratamiento apenas metodológico que se le había dado a dicha correlación mueve a Husserl a volver su atención sobre el problema del conocimiento, no ya para describirlo, sino para solucionarlo. Sin atender a los perspectivismos reduccionistas de las teorías naturalistas, Husserl planteará la correlación bajo la forma del encuentro y mutua afectación entre el sujeto y el mundo, y al emprender su tarea, cada vez verá con mayor claridad cómo un mundo exterior es enfrentado por un mundo interior a través de la puesta en común de una amplia grama de correlaciones desarrolladas en el tiempo e integradas en un mundo vivido como experiencia. Acudiendo al tipo de respuestas que propone la fenomenología, podemos entender ahora la amplia pertinencia que en el mundo de la vida cobran sujeto y objeto.

En “El ‘yo’ en la fenomenología husserliana”, D. Herrera afirma: “¿Qué es el Yo para Husserl? Digámoslo puntualmente y desde ahora: para Husserl no existe un Yo, si por Yo se entiende una entidad -poco importa su naturaleza- que habite nuestro cuerpo como si éste fuese su receptáculo”2. Esta lacónica declaración subsume los alcances de la amplia y quizás inacabada investigación de Husserl en tomo a la conciencia, como consistencia fenomenológica del yo. En Investigaciones lógicas II (1901), Husserl pone en discusión la conciencia, y de paso la vivencia y el yo, según tres conceptos:

a) Como unidad fenomenológico-real de las vivencias del yo;

b) Como percepción interna; c) Como nombre colectivo para toda clase de “actos psíquicos” o “vivencias intencionales”.

El primero de estos conceptos, en virtud del cual Husserl rechaza el yo puro trascendental de Kant, brinda a cambio la ocasión para aceptar y declarar la existencia de un yo empírico y real, evidente y seguro, que se lleva y que se trae como un cuerpo, como una forma o una definición material del sujeto. La precisión de Husserl es, en este sentido, supremamente importante, porque la conciencia no es para él pensamiento pensado, y su constitución radica en sus vinculaciones con la realidad. En el marco de esta discusión, Husserl adelantará la descripción del yo sacada del contenido de la conciencia y la representación del mismo como objeto; “(...) pero en cuanto lo pensamos como objeto -anota Husserl-, hemos dejado de pensarlo como yo. Ser yo no significa ser objeto, sino ser, frente a todo objeto, aquello para lo que algo es objeto"3.

Volviendo con la cita recogida del ensayo de D. Herrera tenemos que, si bien lo expuesto allí llegará a ser la consideración fundamental y definitiva de Husserl en cuanto al yo, cabe decir, la declaración de su inexistencia como cosa real asociada a un cuerpo, en el curso de su obra habrá distintos momentos en los que alternará entre la negación y la aceptación de un yo puro trascendental recogido de la oposición kantiana en contra del empirismo inglés, ya que un yo trascendental (un yo sin propiedades) anunciaría un tipo de respuesta improcedente al problema central de la experiencia humana. A cambio, el yo podría ser una unidad objetiva imputable a cada ser humano en su puesta en relación

1 Daniel Herrera Restrepo, “Qué es la fenomenología”, La persona y el mundo de la experiencia (Bogotá: Universidad de San Buenaventura, 2002) 1-21.

2 Daniel Herrera Restrepo, “El ‘yo’ en la fenomenología husserliana”, América Latina y la fenomenología (México: Universidad Pontificia de México, 1998) 72.

3 Edmund Husserl, Investigaciones lógicas II (Barcelona: Altaya, 1995) 485. 
con el mundo de la vida, pero como comprobación de diferentes estados integrados en él: un yo instintivo o anímico, uno intracorporal, uno personal, y uno más como realización y constitución de la comprensión de su propia relación con el mundo. D. Herrera resalta a continuación el tipo de elección tomada por Husserl a fin de vincular el yo con la vida humana, pues en la conveniencia de situar el yo, de exponerlo y defenderlo como "una representación (una reflexión) anticipante” del mismo, se excusa la tarea de entregarlo a un ejercicio metafísico de autoconciencia.

\section{Descartes y Kant, fuentes del yo en Husserl}

La critica de Descartes al escepticismo, que en las Sextas objeciones y respuestas nos lleva paso a paso al concepto último de 'conciencia pura', realiza el más complejo ejercicio de autognosis cuando, evaluando el papel de la conciencia, declara su separación de toda determinación material; una 'conciencia pura' en la que se escenifica el sujeto activo desde una perspectiva intelectual; el sujeto que piensa, una versión básica y determinante del yo no necesariamente coincidente con el ser humano o con el ser sensible. Siguiendo el método analítico cartesiano se establece de paso la diferencia entre las conciencias puras y separadas, y los denominados por Descartes “animales máquinas", en el sentido en que la plena materialidad ofrece, por su parte, la versión posible de seres automatizados como máquinas, reducidos a una condición animal de la que no alcanzan a sacar provecho ${ }^{4}$.

Cuando hablamos de 'conciencia pura' estamos señalando necesariamente el yo analítico del racionalismo; el mismo que en un "teatro cartesiano” se representa a sí mismo como último bastión de la duda metódica. “(...) pero dudar que pienso no es ya posible, como dudar que soy (...)”, escribe Descartes, quien no recomienda dudar de sí mismo; antes bien, conserva la elección del pensamiento como facultad fundante del sujeto, a la cual ha llegado luego de la descripción analítica de la relación del hombre con la realidad, postulada como el resultado del ejercicio metodológico de invertir en el nivel teórico la formulación que en un plano real ofrecen el cuerpo y la mente, que interactúan, que se corresponden, que se determinan naturalmente.

Como resalta Danilo Cruz Vélez, llevar hasta las últimas consecuencias la opción analítica cartesiana significa declarar, como lo hacen los empiristas ingleses, que sólo una versión empírica del ser humano, un estudio del yo como si fuera una cosa, daría cuenta suya como unidad objetiva. Luego la vía falsa indicada por Descartes impide la intervención del sujeto en la realidad como ser semiente y consciente al mismo tiempo: "Para Descartes -comenta D. Cruz Vélezel ego es res cogitans, substantia cogitans, anima, nombres todos que sólo tienen sentido si se concibe el yo como una cosa. De

4 La que denominamos “versión básica y determinante del 'yo'” significa al menos tres opciones:

a. Mientras que la conciencia pura y separada es autónoma en su ejecución del pensar, los "animales máquinas" no lo son en absoluto; sus actos conscientes, si lo fueren, proceden de un automatismo mecanicista y sensible que no les brindará nunca la opción del pensar.

b. Mientras que las conciencias puras y separadas se afirman en la última comprensión de existencia por el propio efecto del pensar, los “animales máquinas” no advierten nunca que existen, no consiguen realizar esta comprensión básica.

c. Mientras que las conciencias puras y separadas se reconocen como un "yo", los "animales máquinas" están imposibilitados para reconocerse siquiera como individualidad en relación con otras individualidades. 
este modo echa a perder su descubrimiento" ${ }^{\text {. }}$ Efectivamente, no es posible describir un yo elidido del que se abstraen sensaciones y pasiones, ni aún llegando a la última comprensión de su "pensar" como elaboración autónoma y como globalización de su existencia. El empeño de la descripción metafísica cartesiana eludía así el papel de las sensaciones y de las pasiones, desatendiendo en las primeras su papel como primeros intérpretes en la relación del hombre con la realidad, y en las segundas la escenificación en el cuerpo de los contenidos del deseo y los movimientos de la voluntad. De esta manera, la pretensión analítica parece haber encontrado sus propios límites si el concepto del yo surgido de ella no acoge una nueva y determinante instancia de representación: el yo sensiente y pasional enfrentado al mundo.

"Siguiendo el camino inicial de Descartes anota D. Cruz Vélez-, Husserl reconquista en las Ideas $I$ el yo trascendental kantiano que había rechazado en las Investigaciones lógicas"6. Las reglas que operan en la descripción kantiana de la conciencia, reglas a través de las cuales se va de la multiplicidad de las "apercepciones" a un conocimiento no pasivo, posibilitan un "pensar" que, a diferencia del "pensar" expuesto por Descartes, es activo y espontáneo, recreándose de manera continua en su propio ejercitarse como pensar. A partir de esta "reelaboración" de la subjetividad, "reelaboración” del yo, es posible formular por primera vez en filosofía la pregunta “¿Cómo llega el sujeto a conocerse a sí mismo?”, la cual es fundamental y ocupa un espacio central en Kant. Entramos aquí en un momento diferente en el tratamiento del yo, un momento que señala la “apercepción” como directamente vinculada con la autoconciencia trascendental. Pero, ¿cuál es, en este programa, el vehículo del conocimiento que nos acercará de manera más directa a un tratamiento renovado de la subjetividad? En una palabra: la síntesis. Antes que el análisis, la síntesis proporcionará el recurso adecuado a una distinción de la conciencia como producto de la multiplicidad de representaciones dadas en ella. A partir de una síntesis primaria, la realidad nos es dada como respuesta, como coherencia, interpretación y comprensión trascendental, función de organización y definición a partir de la cual declarar el conocimiento como elaboración subjetiva, como versión subjetiva de la realidad, como conciencia propiamente de la realidad.

En este punto particular podríamos abrir la exposición para sopesar distintas versiones acerca del yo y entender ante todo que no se trata ya de entrar en relación con la realidad, de confrontar los móviles de la percepción, sino de distinguir en el término 'apercepción' de Kant la base de una primera respuesta a la pregunta por el conocimiento. Así tenemos:

a) La realidad sintética del sujeto plantea la posibilidad de conectar el yo con la otra realidad, como un acto radical que no reclama una actitud escéptica dirigida a sospechar de su reconocimiento y declaración como entidad básica.

b) De manera opuesta, el método analítico, que vacila en los términos de la ciencia, y que diferencia por actitud el yo y la otra realidad, elude cualquier valoración de la realidad al margen del ingreso sensible en ella.

c) En la decisión que se tome en relación con estas dos opciones se anuncian todas las escuelas posibles de la filosofía: el

\footnotetext{
Danilo Cruz Vélez, Filosofía sin supuestos (Manizales: Editorial Universidad de Caldas, 2001) 134.

6 Cruz Vélez, Filosofía ..., 160.
} 
reduccionismo materialista, el escepticismo, el dualismo, el intuicionismo.

La apercepción kantiana constituye entonces una elaboración, antes que una simple confrontación entre una realidad y unos órganos de la percepción; en la apercepción se señala, inicialmente, no una conciencia del yo, como tal, no una intuición, sino un pensamiento acerca del yo, es decir, una representación, una síntesis, una unidad sintética. “La determinación de mi existencia -escribe Kant-sólo puede tener lugar según la forma del sentido interno, y según la manera particular en que lo diverso que yo enlazo está dado en la intuición interna, y por consiguiente, no me conozco como soy, sino simplemente como ante mí aparezco”7.

La solución del problema del conocimiento -para retomar nuestra exposición- ofrecía a Husserl la ocasión para realizar una crítica del conocimiento sin hacer uso de las ciencias originadas en el pensamiento natural; amarrada a los conceptos de 'naturaleza' y 'experiencia', la actitud natural era retomada por Husserl de la exposición kantiana con el propósito de ver en ella un punto originario en su visión del ser. La diferencia salta a la vista; mientras el sujeto kantiano es, de alguna manera, vacío en sí mismo, es decir, una mera condición formal de la razón, el sujeto husserliano es activo y su análisis revela simultáneamente el sentido del ser del conocimiento como el del ser del objeto. D. Herrera precisa: "Si Kant realiza la síntesis entre la razón y la experiencia, Husserl realiza una racionalización de la experiencia. Si el a priori kantiano sólo es formal, el de Husserl es simultáneamente formal y material. Si Kant responde a su problema con una teoría epistemológica, Husserl, mediante su solución, llega a una teoría sobre la esencia de la conciencia y de la objetividad"8. Lo que se opone aquí, en últimas, son la crítica kantiana y la fenomenología husserliana; oposición que, en palabras de Paul Ricoeur, "debe situarse no en el nivel de la exploración del mundo de los fenómenos, sino en el nivel en el que Kant determina el estatuto ontológico de los fenómenos mismos" ${ }^{\text {" }}$.

\section{El cara a cara D. Cruz Vélez - D. Herrera}

D. Cruz Vélez y D. Herrera exponen en sendos trabajos los pormenores del problema del yo en Husserl; hablamos de "Vicisitudes del yo en Husserl” (1969), de D. Cruz Vélez, y "El yo en la fenomenología husserliana” (1998), de D. Herrera. El conocimiento de la obra de Husserl y de la importancia que cobra en ella el problema del yo, hace que estos trabajos sean inmensamente coincidentes; lecciones de la mejor propedéutica fenomenológica. Si bien los dos trabajos toman rumbos diferentes, ambos siguen los pasos dados por Husserl desde Investigaciones lógicas hasta Ideas II. En su ensayo, D. Cruz Vélez deriva hacia la aplicación del método destructivo del parágrafo 25 de Ser y tiempo, donde Heidegger "pone en marcha la superación de la metafísica de la subjetividad”. D. Herrera se concentra a cambio en los desarrollos de Husserl con respecto al yo que experimenta el mundo, plasmados en Ideas II (edición póstuma de 1952) y en los anexos de Crisis.

A partir de la confrontación que Heidegger hace a Husserl, D. Cruz Vélez interroga:

Emmanuel Kant, Crítica de la razón pura (Buenos Aires: Losada, 1979) 274-275.

8 Daniel Herrera Restrepo, “Un primer esbozo de la fenomenología de Husserl”, Escritos sobre fenomenología, Biblioteca Colombiana de Filosofía (Bogotá: Universidad Santo Tomás, 1986) 200.

9 Paul Ricoeur, “Kant y Husserl”, Praxis filosófica (Cali) 10-11 (número monográfico "Perspectivas de la fenomenología”) (1999): 45-78. 
“¿Dónde debe comenzar el análisis? ¿En el yo o en la existencia? ¿Cuál de estas dos determinaciones del Dasein tiene una preeminencia?” Si bien en la idea de 'vicisitud' había ya un tipo de crítica a las continuas reformulaciones del yo por parte de Husserl, lo que D. Cruz Vélez consigue diferenciar es una limitación metodológica que retiene el tratamiento del yo como subjetividad, sin dar paso a su ejecución como existencia. Hacia el final de su texto, D. Cruz Vélez agudiza la crítica hasta declarar que "la reflexión no puede ser la vía de acceso al yo", que "el yo actual no puede ser objetivado, pues es pura actividad", que "la categoría de identidad no puede servir para determinar el yo", y que "aun cuando esté delimitado por su pasado el yo es libre"10. En estas palabras está distinguiendo D. Cruz Vélez con varias décadas de anticipación uno de los conceptos fundamentales de la etología, la resiliencia, de gran importancia para comprender que los seres humanos no obedecen de manera rígida a las experiencias más difíciles y adversas, conservando a cambio los arrestos necesarios para restituir su yo y para proyectarlo de nuevo en el tiempo.

D. Herrera reconoce igualmente que la concepción del yo en Husserl es paradójica, pero tiene la serenidad suficiente para entender que lo que Husserl emprendió fue una búsqueda pertinaz que lo llevó a su descripción definitiva del "yo puro trascendental", entendido como el sujeto reflexivo involucrado con el mundo, expuesto como cuerpo y proyectado en un tiempo compartido con otros sujetos igualmente reflexivos. D. Herrera no deja de pensar en ese yo que se construye en la vida y en los dramas cotidianos, para acceder al cual se necesita de otros que con sus angustias, éxitos y dificultades aporten los elementos necesarios y constitutivos para que todos tengan un yo. "Al filósofo que desea esclarecer la experiencia humana-afirma D. Herrera- no le queda más remedio que comenzar por la contemplación de su propia existencia. Pero en esta experiencia yo descubro a los otros Egos, porque la estructura típica de mi yo, de mi vida como vida que experimenta el mundo, es la misma estructura de todo yo en primera persona (...). El yo trascendental soy yo, hombre de carne y hueso, que opero en el mundo y que gracias a la reflexión puedo poner delante de mí mismo la totalidad de mi ser operante en un presente viviente que retiene mi pasado y anticipa mi futuro" ${ }^{11}$.

En el capítulo "El yo puro" de Ideas II, Husserl presenta la intencionalidad como dirección de estados mentales hacia el propio yo: "Me hallo -escribe- en el desear atraído por el objeto deseado; estoy dirigido a él, pero de tal modo que aspiro a él, sin alcanzarlo, sin embargo, en el mero desear"12. En ese tender hacia los objetos, en ese realizarse el yo en las cosas en beneficio de ellas y de sí mismo, se encuentran los dos movimientos complementarios de atracción y repulsión como integrados a elecciones y conceptos de las cosas. Como ejecutante, el yo está en la vivencia como entre las mismas cosas, aunque se hunda en ocasiones en la inactividad, como mostrando que hay una estructura que realza a la vez una actividad y una latencia. D. Cruz Vélez plantea que el “yo personal” descrito por Husserl allí no es un mero ingrediente de la naturaleza, sino un miembro de comunidades personales como la amistad, el matrimonio, la familia, las clases sociales, la Iglesia, el Estado: "La causalidad que determina a este yo -anotano es la causalidad natural, sino la motiva-

Cf. Cruz Vélez, Filosofía ..., 185-186.

11 Herrera Restrepo, “El ‘yo' ...”, 82-83.

12 Edmund Husserl, Ideas relativas a una fenomenología pura y una filosofía fenomenológica, vol. II de Investigaciones fenomenológicas sobre la constitución (México: UNAM, 1997) 134. 
ción. Es decir, lo determinante en él no son causas naturales extemas o internas, como ocurre en el yo intracorporal y en el yo anímico, sino, por ejemplo, ideas, creencias, mandatos, teorías, fines, vivencias estéticas, morales o religiosas (...)”13. Queda señalado así cómo a ese "yo personal”, antesala del "yo puro trascendental", le corresponde a continuación una percepción de sí mismo, tanto como un recuerdo de sí y una fantasía de sí, para ser un yo que se capta como temporalmente duradero.

El "yo del último Husserl”, como lo denomina D. Herrera en "Nosotros y la fenomenología", ${ }^{14}$ ha ingresado en la temporalidad con las dinámicas propias de un ser que vive el tiempo aproximándolo a sí, ingresando y proyectándose en el tiempo. Así, si el "yo pienso" no es ahora suficiente ni en un plano metodológico, porque no señala ningún tipo de relación con el tiempo, a cambio, una amalgama de decisiones, como "yo puedo”, “yo quiero”, “yo debo”, plantea la tarea de la ejecución y representación de todos los entreactos entre el sujeto y el mundo. Localizado el yo como función en el tiempo, como estructura de la temporalidad, pleno de opciones y compromisos, indiferente a su autojuzgamiento como mejor o peor, guiado por su propio proyecto de autorreflexión, no tenemos otra opción que reconocerlo como integrado en el mundo de la vida que le ofrece el tiempo.

\section{El desafío de la filosofía como ciencia estricta (rigurosa)}

En palabras de D. Herrera, el artículo "La filosofía como ciencia estricta”, publicado por Husserl en la revista Logos $(1910)^{15}$, "puede ser considerado como un verdadero Manifiesto", es decir, como una clara declaración de principios que lleva de fondo el propósito de ofrecer una nueva definición de la filosofía. La orientación que Husserl consigue dar al pensamiento filosófico durante el siglo XX así lo demuestra, motivando, sin duda, una redefinición de la filosofía desde los parámetros de la sensibilidad, la rigurosidad y la profundidad. Un "manifiesto", sí, por el impacto producido, por su resonancia. D. Cruz Vélez resalta igualmente cómo los estudios e investigaciones de Husserl "lo llevaron a una reforma radical de la filosofía”, sin comprometer en ello una decisión en tomo a innovaciones o mejoramientos de la filosofía, sino del ejercicio mismo, discutido ahora bajo el controvertido rótulo de "ciencia estricta", fundamentadora de las disciplinas científicas. Pero, ¿qué mérito tiene para la descripción del pensamiento husserliano que estas dos voces, como figuras del coro, insistan una en el tratamiento de la filosofía como "ciencia rigurosa" (D. Herrera), otra como "ciencia estricta” (D. Cruz Vélez)? La diferencia entre uno y otro término, entre una y otra valoración resalta más allá de la simple decisión de la traducción. Veamos:

a) Si lo "estricto" señala aquello ajustado a la ley y, como tal, que no admite interpretación, la filosofía sería para Husserl estricta por virtud de su ajuste a la ciencia, que le reclama exactitud, correspondencia fiel con los objetos puestos bajo su consideración.

b) A cambio, la elección del término 'riguroso’ permite recoger una precisión adi-

13 Cruz Vélez, Filosofía ..., 165.

14 Cf. Daniel Herrera Restrepo, “Nosotros y la fenomenología”, América Latina y la fenomenología (México: Universidad Pontificia de México, 1998).

15 Edmund Husserl, "Philosophie als strenge Wissenschaft”, Logos: Intemationale Zeitschríft für Philosophie der Kultur, I. 3 (1910/1911): 289-341. 
cional; es riguroso un saber que es inflexible y que desatiende la dispersión de los naturalismos, historicismos y psicologismos. En aras de la minuciosidad e intensidad en los procedimientos de observación y evaluación, la filosofía reclama rigurosidad.

Valorando el tratamiento de la filosofía como “ciencia estricta”, privilegiamos la estrechez deliberativa de las ciencias, ajenas a la interpretación; esta limitación lleva a D. Cruz Vélez a plantear la enconada conclusión:

"La filosofía no es una ciencia, porque su idea de la verdad no corresponde a la de la ciencia (...). La filosofía no es una ciencia, porque ella no puede fundamentar sus juicios como las ciencias (...). La filosofía no es una ciencia, porque no puede alcanzar, como las ciencias, juicios válidos y para siempre (...). La filosofía no es una ciencia, porque no puede alcanzar, como las ciencias, juicios válidos para todo el mundo”16.

Por su parte, D. Herrera está viendo los beneficios de la rigurosidad de la filosofía como eliminación de las tendencias naturalistas, historicistas y psicoligistas que desvirtúan el verdadero interés de la filosofía, llamado a establecer una relación correcta con los objetos. No obstante, la discusión de fondo en ambos trabajos es, por supuesto, el tratamiento desafiante de Husserl en relación con la filosofía, no sólo al declararla "ciencia” fundamentadora de las demás ciencias, sino al proyectarla como conjunto de verdades organizadas sistemáticamente, espacio en el que interviene con claridad la salvedad planteada por D. Herrera: "La filosofía, ciencia de la conciencia, no podrá ser una ciencia 'exacta' ya que la temporalidad es algo esencial de las vivencias. Por consiguiente, éstas no pueden ser 'fijadas' para ser sometidas a 'medidas exactas'. Pero la filosofía sí puede ser rigurosa en la medida en que sus verdades son objetivas y gozan por lo mismo de validez absoluta” ${ }^{17}$.

El momento es propicio para volver sobre la posición de Husserl en contra de quienes sostenían tesis acerca de la naturalización radical de la conciencia; Husserl denunció como idealistas, objetivistas e ingenuos a los promotores de dichas tesis; lo primero por construir teorías que proclaman a la vez valores o normas prácticas; lo segundo por creer cumplida su tarea por medio de la ciencia de la naturaleza; lo tercero por sobreentender que las cosas sencillamente son. Nuestra pregunta es ahora: ¿Se aplica la crítica husserliana a quienes exponen hoy en términos similares tesis en tomo a la naturalización de la mente? ${ }^{18}$. En el orden psíquico del yo, lo natural está dado como vivencia que involucra la experiencia y el tratamiento del cuerpo, lo que dificulta su descripción, pues si cada acto de conciencia es "conciencia de...”, su estudio comprende no sólo objetividad sino también significación y realización. En esta afirmación está puesta en cuestión toda actitud naturalizadora de la conciencia que no tenga presente la unidad y complejidad de los eventos mentales, toda filosofía de la mente que focalice su interpreta-

16 Cruz Vélez, Filosofía ..., 234-235.

17 Daniel Herrera Restrepo, “Los orígenes de la filosofía como fenomenología”, Escritos sobre fenomenología, Biblioteca Colombiana de Filosofía (Bogotá: Universidad Santo Tomás, 1996) 97.

18 Los filósofos colombianos Juan José Botero, Jaime Ramos y Alejandro Rosas caracterizan la naturalización de la mente en los siguientes términos: "La ciencia cognitiva, en cuento tal, intenta constituirse como ciencia natural de la mente. Su propósito puede entenderse como el de la construcción de una teoría de los fenómenos mentales que permita elaborar explicaciones que sean aceptables para un enfoque naturalista de las propiedades de la mente”. Varios, Mentes reales: La ciencia cognitiva y la naturalización de la mente (Bogotá: Siglo del Hombre/ Universidad Nacional de Colombia, 2000) 11. 
ción de lo humano o del yo en la descripción del cerebro, sin tener presentes los niveles de significación que desempeña la mente en relación con el mundo. No hay aquí, como se ve, una descalificación rotunda de la naturalización, sino una advertencia importante. Tras estas consideraciones, Husserl resalta el principio de rigor científico requerido para que, ni el trabajo de observación, ni los de descripción y proposición de teorías, desvíen en las insuficiencias de todo positivismo, que desconocen la realización de una humanidad auténtica.

D. Herrera ha vuelto de manera insistente sobre estos asuntos intentando, no ya responder al desafío husserliano de la filosofía como ciencia estricta, sino localizar en el yo la advertencia que permite aceptar nuevos planteamientos acerca de la naturalización de la conciencia, como los de Marvin Minsky, con su teoría representacional de la mente, los de Hubert Dreyms, asociados a una crítica fenomenológica de la inteligencia artificial, y, a partir de Dreyms, los del fenomenólogo colombiano Germán Vargas Guillen, de quien reconoce: "sin abandonar el marco de la fenomenología eidética y sin recurrir a los datos de las ciencias naturales, investiga la posibilidad de una 'naturalización de la conciencia' por los caminos de la Inteligencia Artificial”"

Lo que se ha ganado con la conferencia de Husserl de 1910 no es, finalmente, la ocasión para delimitar con claridad el trabajo y el objeto de estudio de las ciencias como opuesto al de la filosofía, sino todo lo contrario, la advertencia de un encuentro de intereses, de una empresa común que debe caracterizarse por el rigor y la claridad en el tratamiento de la experiencia humana. Desde una perspectiva empírica de las ciencias, la fenomenología es vista como ciencia especulativa de la subjetividad, y algunos de sus aspectos aparecen como no científicos, lo que no obsta para que en el contexto de avance de las ciencias cognitivas uno de sus propósitos consista, en lo que se denominaría "fenomenología experimental" o "fenomenología empírica”, en hacer posible fuera de la fenomenología trascendental problemas como el análisis de la temporalidad de la conciencia y la descripción de diferentes actos de conciencia como la atención, la memoria, la percepción.

\section{Otros encuentros}

Para D. Cruz Vélez, ‘constitución’ es el nombre con el que aparece en Husserl el problema metafísico de la explicación del ser de los entes; en este sentido, la investigación fenomenológica adelantada por Husserl en Ideas II se propone mostrar cómo los objetos se constituyen por obra de las intervenciones del sujeto en ellos, marco en el cual se plantea, precisamente, el problema del "yo puro". Para D. Herrera, por su parte, "constituir” es ver en la reflexión cómo los entes se me hacen presentes y se interrelacionan a su vez según determinadas formas, y ver simultáneamente cómo esas formas o modos dependen de la institucionalidad, de los proyectos, de los modos a través de los cuales me dirijo a ellos. Entre facticidad y subjetividad se construye el sentido del mundo y de sus entes, que se refieren a la constitución misma del yo (con su cuerpo o sin su cuerpo) como realidad; “constituir” es así asistir en la reflexión a la génesis de un sentido.

Como ésta, en repetidas ocasiones D. Cruz Vélez y D. Herrera adelantan indagaciones paralelas sobre asuntos husserlianos; una de ellas, decisiva para la comprensión de los problemas del yo y del conocimiento en Husserl, recae sobre los pormenores de las

19 Herrera Restrepo, “Qué es ...”, 17. 
actitudes natural y filosófica señaladas por Husserl. En el capítulo XII de Filosofía sin supuestos, D. Cruz Vélez ve la actitud natural del sujeto como origen de su relación con el conocimiento, y el mundo de la actitud natural como el ámbito donde hombres de la actitud natural representan, juzgan, sienten y quieren en esa actitud: “(...) la naturaleza que el hombre recibe por el mero hecho de nacer-comenta-. Ella determina la actitud natural, en la cual lo que se da como verdadero no es más que ilusión”². D. Cruz Vélez reconoce igualmente el papel de la intencionalidad en la actitud natural, actitud que confronta al yo como ejecutor de intenciones, retenciones y protensiones que declaran en él su vocación y sus posibilidades de cara a otra actitud, la filosófica reflexiva.

A su turno, comentando las dos actitudes o “dimensiones” diferentes del pensamiento, en "Un primer esbozo de la fenomenología de Husserl” (1969), D. Herrera llega a precisiones en turno al yo que lo apartan de su tratamiento, o como simple cosa, o como inmanencia. Para D. Herrera, en aquel texto la teoría husserliana de la "reducción” ha permitido descubrir que ninguno de estos dos yo está presente en la intuición pura, que el descubrimiento de Husserl de la inmanencia pura y su trascendencia entendidos como punto de partida del problema del conocimiento, constituye el contacto, la puesta en relación entre el sujeto y el objeto, fuera de los términos de lo enigmático. De las exposiciones de los dos filósofos recogemos finalmente lo siguiente:

a) En la idea de toda actitud advertimos inicialmente, para utilizar un término crucial en Husserl, la “reducción” de la potencia al acto, en el sentido en que la actitud es ya exteriorización de una posibilidad que pone al yo en función de algo, en función de su estar en el mundo, en función de su naturaleza y de su posterior comprensión de la misma en la actitud filosófica. La actitud le da constitución al sujeto en tanto que natural, quien, de no ofrecerse así, mantendría desligado de su representación y de su conocimiento.

b) La actitud es una puesta en ejercicio, un acortamiento de la distancia en relación con la actuación; así, la actitud natural pone en ejercicio la naturaleza inmanente del sujeto con toda su viveza, en tanto que la actitud filosófica hace efectivo el ponderado de la razón, cuando el sujeto actúa sobre sí mismo a través de la observación, la indagación y la reflexión.

c) En el sentido más corriente, la actitud expone ese estado previo en el que se encuentra un sujeto que se dispone a dar una respuesta; la dificultad en este caso señala la imposibilidad de delimitar y diferenciar dicho estado previo, que no era, como tal, otro estado, porque tanto la respuesta como el estado previo pertenecen a un mismo estado natural.

\section{Colofón}

Tanto insiste D. Herrera en la soberana intuición de Husserl de 1898, según la cual entre el sujeto y el objeto hay correlaciones, y cómo a partir de ahí todo su trabajo consiste en desarrollar esa intuición dando forma a la fenomenología, que nos entra la sospecha de la movilización de un eterno retorno en relación con aquella intuición, vivida veinticinco siglos atrás en Benarés, en el Nepal, por el príncipe Gautama Buddha, quien tuvo una intuición similar, acaso más determinante aún, de que todas las causas y todos los efectos tienen relación, de que todo depende y todo pertenece a todo, que todo es algo para todo, así en el tiempo como en el espacio.

20 Cruz Vélez, Filosofía ..., 247. 